\title{
INFLUÊNCIA E INTERFERÊNCIA: CRUZANDO DOIS PARADIGMAS DE PRIMAÇÃO AFECTIVA
}

\author{
Marília Prada ${ }^{1}$ \\ Teresa Garcia-Marques ${ }^{2}$
}

Resumo: Neste artigo, focamos o fenómeno de primação afectiva (impacto de activação prévia de valência afectiva em processamento subsequente), contrastando dois dos principais paradigmas experimentais que lhe dão suporte: interferência e influência. Em dois experimentos, analisámos os dados relativos ao enviesamento de resposta avaliativa e aos tempos de reacção. O Experimento 1 mostra que a apresentação de uma palavra valenciada facilita (interfere) respostas subsequentes congruentes (incongruentes). Analisando também o tipo de resposta dada pelos participantes, verificou-se um maior número de erros nos ensaios incongruentes. $\mathrm{O}$ Experimento 2 demonstra o efeito de influência em que imagens-primo induzem julgamentos avaliativos de alvos, previamente neutros, congruentes com a sua valência. $\mathrm{O}$ estudo dos tempos de reacção neste paradigma revelou que primos negativos induziam maior rapidez de resposta. Vantagens e desvantagens de ambos os paradigmas são discutidas relativamente à sua relevância para o efeito de primação e para a sua aplicabilidade enquanto medidas implícitas de atitudes.

Palavras-chave: Primação afectiva, interferência, influência.

Influence and Interference: Combining two affective priming paradigms (Abstract): In this paper, we investigate affective priming phenomenon (impact of a previously activated valence in subsequent processing) by contrasting two of the main experimental paradigms used in this domain: interference and influence paradigms. In two experiments, data referring both to the evaluative response bias and response times were analysed. Experiment 1 demonstrates that the presentation of a valenced prime-word facilitates (interferes) processing of a subsequent congru-

\footnotetext{
${ }^{1}$ ISCTE, Instituto Universitário de Lisboa / UIPCDE, ISPA, Instituto Superior de Psicologia Aplicada, Lisboa.

2 ISPA, Instituto Superior de Psicologia Aplicada, Lisboa.

Nota dos Autores: Este trabalho teve os apoios das bolsas: POCI 2010, BD atribuída a Marília Prada e PTDC/PSI/64944/2006, atribuída a Teresa Garcia-Marques pela Fundação para a Ciência e Tecnologia. A correspondência sobre este artigo pode ser dirigida a mprada@ispa.pt, e gmarques@ispa.pt ou Instituto Superior de Psicologia Aplicada - Rua Jardim do Tabaco, 34, 1149-041 Lisboa, Portugal. Tel. +351 21881 1700; Fax: +351 21 8860954.
} 
ent (incongruent) stimulus. The analysis considering the type of response given by the participants reveals a higher number of errors for incongruent trials. Experiment 2 demonstrates an influence effect given that judgments of target-images portraying neutral objects are influenced in the direction of the valence activated by the primes. Response time analysis in this paradigm revealed faster responses when the primes were negative. Advantages and disadvantages associated in both types of paradigms are discussed considering their relevance to affective priming effect and their applicability as attitudes implicit measures.

Keywords: Affective Priming, interference, influence.

Bebés. Morangos. Dançar. Flores. Gatinhos. Namorar. Pastéis de Nata. Sorrisos. Festa. Neste momento estará a pensar o que estes poderão ter em comum. De facto, semanticamente não há muito que os relacione. No entanto, a mera leitura destas palavras pode ser suficiente para o fazer esboçar um sorriso e/ou activar uma série de boas recordações (por exemplo, aquela visita a Belém e o cheiro da canela nos pastéis mornos e estaladiços). Por agora já deve ter reparado que uma das características partilhadas por estas palavras é serem associadas na nossa mente a algo positivo. É exactamente dos efeitos deste tipo de activação que trata a investigação da primação afectiva (affective priming). Em termos gerais, o estudo do fenómeno de primação centra-se na investigação do impacto da activação temporária de representações mentais pelo contexto em diversos fenómenos psicológicos incluindo a percepção, avaliação, motivação e comportamento (Bargh \& Chartrand, 2000). Se a representação activada for de natureza afectiva ou avaliativa, tal impacto designa-se por efeito de primação afectiva. Por outras palavras, o fenómeno da primação afectiva refere-se à influência não intencional de uma primeira resposta avaliativa a um dado estímulo (i.e., prime ou estímulo-primo, por exemplo "pastel de nata") no processamento subsequente de um outro estímulo (i.e., target ou estímulo-alvo) (Klauer, 1998).

A popularidade que a primação, enquanto metodologia, alcançou na literatura (para revisão, ver Bargh, 2006) baseia-se no pressuposto de que esta possibilita uma forma de acesso privilegiado aos mecanismos e estruturas de conhecimento do processamento automático de informação que se supõe constituírem o substrato da cognição social (e.g., Banse, 2001). Dada a sua grande flexibilidade (uma vez que tanto a relação entre ambos os estímulos como a natureza de processamento do alvo pode ser manipulada) tem sido aplicada à investigação em diversos domínios (De Houwer, Hermans, \& Eelen, 1998). Por exemplo, os efeitos de primação têm sido úteis 
para investigar a natureza da memória associativa, estudar processos emocionais ou diagnosticar atitudes e preconceitos (para revisão, ver Fiedler, 2003). Especificamente, a primação afectiva tem sido a principal ferramenta para a investigação de processos avaliativos automáticos, assumidos como transversais à existência humana (e.g., Fergunson \& Bargh, 2003). De facto, a investigação tem contribuído para o estabelecimento da noção do percipiente social enquanto "avaliador", dada a importância e variedade de situações que requerem a determinação de valência (Winkielman, Schwarz, Fazendeiro, \& Reber, 2003). Sabemos, por exemplo, que mesmo estímulos novos, cujo significado é desconhecido (e.g., palavras estrangeiras), são passíveis de suscitar reacções avaliativas (Duckworth, Bargh, Garcia, \& Chaiken, 2002). Por outras palavras, tais estímulos são automaticamente avaliados.

Neste artigo abordaremos o fenómeno de primação afectiva em dois paradigmas distintos: "interferência" e de "influência" (Garcia-Marques, 2005; Prada \& Garcia-Marques, 2006; para uma distinção semelhante ver Gawronski, Deutsch, Le Bel, \& Peters, 2008). Procuraremos tornar claro o fenómeno que os une e as características que os diferenciam. Em dois estudos distintos utilizaremos cada um destes paradigmas, com estímulos de natureza diversa, como palavras e imagens, demonstrando a generalidade do fenómeno e a sua manifestação aos níveis supra e subliminar.

\section{Primação Afectiva: Fenómeno e paradigmas}

A primação afectiva refere-se ao fenómeno de que o processamento de um estímulo valenciado (e.g., a palavra "amor") é facilitado quando é precedido por um outro estímulo da mesma valência (e.g., "felicidade") comparativamente a ser precedido por um de valência oposta (e.g. "morte"). A valência previamente activada pode também influenciar a natureza do julgamento subsequente, particularmente quanto tal julgamento é referente a um alvo neutro ou ambíguo em termos avaliativos.

Tais impactos da activação da valência afectiva de um estímulo (ou seja, a propriedade através da qual este é percebido como bom/mau ou positivo/negativo) podem ser detectados através do recurso a vários paradigmas experimentais (e.g., paradigmas afectivos de Stroop e de Simon; para revisão, ver Musch \& Klauer, 2003). Os mais preponderantes na literatura podem ser classificados, com base no padrão de resultados tipicamente encontrados, como sendo de "interferência" ou de "influência" (Garcia-Marques, 2005; Prada \& Garcia-Marques, 2006). Ou seja, enquanto que o primeiro se centra primordialmente ao nível de diferenças na latência de resposta aos estímulos-alvo, o segundo focaliza diferenças em termos do conteúdo de tais respostas. 
O paradigma de interferência ${ }^{3}$ consiste numa adaptação do utilizado para a investigação de efeitos de primação semântica (para revisão, ver Neely, 1991). Uma das premissas-base é de que a activação de um determinado conteúdo semântico exercerá impacto ao nível do desempenho relativo a um conteúdo subsequente. Por exemplo, a experiência recente com uma palavra tende a facilitar o seu processamento na próxima ocorrência (e.g., Abrams, Klinger, \& Greenwald, 2002). Tipicamente, num estudo desta natureza, cada ensaio envolve a breve apresentação de dois estímulos: uma palavra-primo e outra alvo. O participante é instruído para responder apenas ao alvo, ignorando a palavra-primo, cuja presença é, frequentemente, justificada por uma alegada necessidade de dificultar a tarefa. Quando ambos os estímulos são associados semânticos (e.g., "médico"/ "enfermeira") o desempenho é, habitualmente, facilitado: as respostas aos alvos são mais rápidas e, frequentemente, mais correctas do que no caso de inexistência de qualquer relação semântica (e.g., "pão"/ "enfermeira") (e.g., Meyer \& Schvaneveldt, 1971; Neely, 1976, 1977; Balota \& Lorch, 1986, Henik, Nissimov, Priel, \& Umansky, 1995), sendo que estes efeitos podem mesmo ser obtidos a nível subliminar (e.g., Draine \& Greenwald, 1998).

A versão afectiva deste paradigma envolve a apresentação de estímulos (quer alvos, quer primos) com uma valência afectiva associada, sendo os participantes instruídos apenas para avaliar os alvos, classificando, por exemplo, o seu significado como positivo ou negativo. O padrão de resultados típico é a facilitação de desempenho (i.e., respostas mais rápidas e com menos erros) aquando da congruência avaliativa entre ambos os estímulos (e.g. "morte"/ "agressivo"). Pelo contrário, quando os estímulos não partilham a mesma tonalidade afectiva (e.g. "rosa"/ "agressivo") verifica-se que a interferência no desempenho dos indivíduos é antes de carácter inibitório. Fazio e colaboradores (1986) desenvolveram este paradigma com o intuito de explorar em que medida a mera apresentação de um dado estímulo facilitaria a latência do julgamento relativo à conotação afectiva de um outro subsequente. Os resultados encontrados sugerem a existência do efeito de congruência afectiva que descrevemos anteriormente, tendo suscitado um elevado número de replicações (e, evidentemente, extensão das evidências inicialmente demonstradas), reafirmando-se a sua robustez e utilidade na investigação de fenómenos do foro afectivo (e.g., Bargh, Chaiken, Govender, \& Pratto, 1992; Chan, Ybarra, \& Schwarz, 2006; ver Fazio, 2001 e Klauer \& Musch, 2003, para revisão).

\footnotetext{
${ }^{3}$ Nota: ainda que os efeitos evidenciados por outros paradigmas (como os de Simon e de Stroop afectivos) expressem também interferência no desempenho (Simon \& Berbaum, 1990), no presente artigo entende-se por paradigma de interferência o utilizado na linha de investigação de Fazio, Sanbonmatsu, Kardes e Powell (1986).
} 
O paradigma de influência, por sua vez, envolve apenas a apresentação de estímulos-primo com uma valência afectiva associada, sendo os alvos tipicamente neutros ou ambíguos. Mais uma vez, os participantes são instruídos para apenas efectuar julgamentos acerca dos alvos, verificando-se que tal avaliação é influenciada pelos estímulos que a precedem (i.e., primos), em geral no sentido da valência activada. Ou seja, um alvo que havia sido pré-testado como neutro, será percebido como mais positivo (ou negativo) se for precedido por um estímulo positivo (ou negativo). Por exemplo, nos estudos de Murphy e Zajonc (1993), os participantes eram instruídos para avaliar em que medida gostavam de um conjunto de caracteres chineses (caracterizados pela sua neutralidade ou ambiguidade). Cada um destes caracteres era precedido pela apresentação da imagem de uma face a expressar felicidade (i.e., primo positivo) ou raiva (i.e., primo negativo), verificando-se que os símbolos precedidos pela face "feliz" eram preferidos relativamente aos primados negativamente.

Também este paradigma foi alvo de diversas replicações e adaptações. Por exemplo, Banse (1999; 2003 para revisão) adaptou-o para o domínio das relações interpessoais, visando investigar a possibilidade de existência de uma resposta avaliativa automática, quer a amigos, quer a parceiros românticos. Concretamente, em vez de fotografias ilustrativas de expressões faciais, os participantes eram expostos a breves apresentações de fotografias dos seus parceiros (ou amigos próximos) previamente à avaliação do conjunto de caracteres chineses. Como esperado, os caracteres precedidos por tais estímulos eram avaliados mais positivamente do que os não haviam sido primados.

A descrição que apresentamos corresponde apenas às características de base de cada um dos paradigmas (ver Tabela 1), sendo que cada adaptação poderá possuir particularidades específicas.

\section{Generalidade do efeito de Primação Afectiva e Automatismo}

Os efeitos de primação afectiva têm sido demonstrados com uma elevada diversidade, quer de objectos atitudinais (ou estímulos), quer de tarefas experimentais, tais como: a) decisão avaliativa (e.g., Fazio et al., 1986; Klauer, Roßnagel, \& Musch, 1997); b) decisão lexical (e.g., Hermans, Smeesters, De Houwer, \& Eelen, 2002; Wentura, 1998) ou c) tarefa de pronunciação (e.g., De Houwer, Hermans, \& Spruyt, 2001; De Houwer \& Randell, 2002, 2004; Spruyt, Hermans, De Houwer, \& Eelen, 2002).

Entre os diferentes tipos de estímulos que têm sido utilizados, contam-se: palavras (e.g., Bargh et al., 1992; Chaiken \& Bargh, 1993); não-palavras às quais é experimentalmente associado um significado afectivo (e.g., estudo da tradução de alegadas palavras turcas numa fase inicial do 
estudo - De Houwer et al., 1998); fotografias complexas (e.g., Hermans, Spruyt, De Houwer, \& Eelen, 2003); desenhos simples (e.g., Giner-Sorolla, Garcia, \& Bargh, 1999); ou mesmo odores (Hermans, Baeyens, \& Eelen, 1998). Sublinha-se que não é necessário que ambos os estímulos (i.e., primos e alvos) partilhem a mesma modalidade. A característica-chave é a valência dos estímulos, independentemente da correspondência em termos da sua forma. Por exemplo, uma fotografia pode servir para primar uma palavra-alvo ou vice-versa (e.g., Lamote, Hermans, Baeyens, \& Eelen, 2004; Hermans et al., 1998; Spruyt, et al., 2002).

Tabela 1. Síntese dos paradigmas de interferência e influência

\begin{tabular}{|c|c|c|}
\hline & Interferência & Influência \\
\hline Estímulos-Primo & Valenciados & Valenciados \\
\hline Estímulos-Alvo & Valenciados & Neutros ou ambíguos \\
\hline Resposta & $\begin{array}{l}\text { Dicotómica } \\
\text { (e.g., Positivo vs Negativo) }\end{array}$ & $\begin{array}{l}\text { Escala } \\
\text { (e.g., "Gosto Pouco } 1 \ldots 5 \\
\text { Gosto Muito") }\end{array}$ \\
\hline $\begin{array}{l}\text { Variável Depen- } \\
\text { dente }\end{array}$ & $\begin{array}{l}\text { Tempo de Resposta ao Alvo } \\
\text { Proporção de Acertos }\end{array}$ & Julgamento avaliativo \\
\hline $\begin{array}{l}\text { Detecção do Efei- } \\
\text { to }\end{array}$ & $\begin{array}{l}\text { Comparação entre ensaios } \\
\text { congruentes (facilitação) e } \\
\text { incongruentes (inibição) }\end{array}$ & $\begin{array}{l}\text { Mudança na avaliação do } \\
\text { alvo, habitualmente na direc- } \\
\text { ção da valência activada }\end{array}$ \\
\hline
\end{tabular}

O facto do efeito de primação afectiva ser saliente face a tal variado espectro de objectos e manipulações experimentais tem sido assumido na literatura como evidências que suportam a noção de generalidade do efeito. Mais ainda, tendo em conta os critérios que definem um processo automático com base na ausência de consciência ou atenção, eficiência, intenção e controlo (Bargh, 1994), tem sido proposto que os processos subjacentes aos efeitos de primação afectiva são desta natureza. Isto porque tais efeitos assumem uma latência reduzida, são de curta duração, não parecem depender de um objectivo avaliativo explícito nem da presença de amplos recursos cognitivos, e podem mesmo ser observados com apresentações subliminares (i.e., sob condições que previnem a percepção da sua presença, como tempos de apresentação muito breves) dos estímulos-primos (para revisão ver Hermans, De Houwer, \& Eelen, 2001). 


\section{Mecanismos explicativos dos efeitos de Primação Afectiva}

Existem essencialmente duas perspectivas explicativas dos efeitos de primação afectiva: mecanismo de difusão da activação (e.g., Fazio, 2001; Fazio et al., 1986) e de competição de resposta (e.g., Hermans, De Houwer, \& Eelen, 1996; Klauer, 1998; Klauer et al., 1997; Klinger et al., 2000; Wentura, 1999). Ambas concordam que o ponto de partida é a activação automática da avaliação associada ao objecto primado. Pressupõe-se que tal activação facilita a codificação de alvos afectivamente congruentes (de acordo com o mecanismo de difusão de activação) ou activa uma tendência de resposta inicial que pode ser compatível ou não com a tendência de resposta activada pelo alvo (segundo o mecanismo de competição de resposta). Embora esta questão esteja ainda longe de ser consensual, tem sido sugerida a possibilidade de os efeitos observados neste tipo de paradigmas não derivarem de um único mecanismo, mas serem antes determinados por múltiplos mecanismos como os acima apresentados (para revisão, ver Wittenbrink, 2007).

À luz da importância que o estudo do fenómeno de primação afectiva tem assumido na literatura, é relevante a compreensão dos paradigmas que o sustentam. Neste artigo, apresentamos dois estudos que têm por base os dois principais paradigmas utilizados para a sua investigação - o paradigma de interferência (Experimento 1) e o de influência (Experimento 2). Como forma de os contrastar, propomos a utilidade da exploração dos tempos de resposta e dos julgamentos avaliativos em ambos os paradigmas enquanto potenciais indicadores da verificação deste tipo de efeitos. Ou seja, nos nossos estudos, exploraremos, quer o conteúdo dos julgamentos (i.e., se a resposta ao alvo foi correcta ou não) no paradigma de interferência (para além da análise dos tempos de resposta), quer os tempos de resposta no paradigma de influência (para além dos julgamentos avaliativos).

\section{Experimento 1}

Este experimento faz uso do paradigma de primação afectiva de interferência, baseando-se no procedimento desenvolvido por Fazio e colaboradores (1986). Uma diferença relevante é que, neste experimento, optámos por apresentações únicas de cada alvo (em vez de repetidas por bloco de ensaios), o que tem a vantagem de reduzir substancialmente a duração de aplicação do paradigma. Em termos de hipóteses, esperava-se replicar o efeito de interferência, pelo que, no caso de ensaios incongruentes (i.e., a valência do estímulo-primo difere da do alvo), o desempenho seria dificultado (i.e., respostas menos rápidas) comparativamente aos ensaios con- 
gruentes (i.e., ambos os estímulos partilham a mesma valência). Adicionalmente, e dado que a acuidade de resposta constitui também um indicador de facilitação de desempenho ${ }^{4}$, espera-se que os ensaios congruentes exibam maior proporção de acertos do que os incongruentes. Ou seja, é mais provável um erro no julgamento de valência do alvo quando o estímulo que o precede é da valência oposta (e.g., responder que "fada" é negativo se primada por "doente").

\section{Participantes e Delineamento}

Participaram voluntariamente, mediante crédito parcial numa disciplina do curso de Psicologia, 59 estudantes $(81,4 \%$ do género feminino; $M_{\text {idade }}=25,2$ anos, d.p. $\left.=6,2\right)$. O delineamento experimental era constituído pelos seguintes factores intra-sujeitos: 2 (valência-primo: negativo; positivo) x 2 (valência-alvo: congruente, incongruente).

\section{Material}

O conjunto de palavras-primo era constituído por adjectivos pré-testados como familiares (Prada \& Silva, 2008), diferindo apenas no respeitante à sua valência: 10 negativos $\left(M_{\text {Valência }}=1,8, d . p .=0,22 ; M_{\text {Familiarida- }}\right.$ de $=5,2, d . p .=0,18)$ e 10 positivos $^{5}\left(M_{\text {Valência }}=6,1 ; M_{\text {Familiaridade }}=5,8\right)$. Os estímulos-alvo, por sua vez, consistiam em palavras concretas, de familiaridade média, variando também em termos da sua valência (Garcia-Marques, 2003): 10 negativas $\left(M_{\text {Valência }}=2,4, \quad d . p .=0,43 ; \quad M_{\text {Familiaridade }}=4,0\right.$, d.p. $=0,30)$ e 10 positivas $^{6}\left(M_{\text {Valência }}=5,6, d . p .=0,22 ; M_{\text {Familiaridade }}=4,4\right.$, d.p. $=0,39)$.

\footnotetext{
${ }^{4}$ É pouco comum a análise da proporção de respostas certas por, frequentemente, se verificar um efeito de tecto a este nível - por exemplo, Fazio e colaboradores (1986) referem erros médios inferiores a $2 \%$. Tal efeito poderá dever-se ao facto de alguns paradigmas envolverem múltiplas apresentações dos mesmos alvos (emparelhados com diferentes tipos de primos em diferentes blocos).

${ }^{5} \mathrm{O}$ sub-conjunto de estímulos-primo negativos era composto pela seguintes palavras: "idiota", "ridículo", "sujo", "falso", "estúpido", "irritante", "doente", "pobre", "mau" e "triste" e os positivos por: "lindo", "suave", "social", "original", "terno", "precioso", "alegre", "perfeito", "excitante" e "atraente".

${ }^{6} \mathrm{O}$ sub-conjunto de estímulos-alvo negativos era composto pela seguintes palavras: "ranho", "masmorra", "entulho", "avalanche", "vampiro", "enxofre", "arpão", "betão", "flecha" e "fardo" e os positivos por: "teleférico", "duna", "trevo", "farol", "esmeralda", "lírio", "bússola", "gôndola", "harpa" e "fada".
} 


\section{Procedimento}

Os participantes eram convidados a participar num estudo sobre "avaliação automática e espontânea de palavras" realizado com suporte informático (programa E-prime). A tarefa destes era a de avaliar, o mais rapidamente possível, a palavra que ficasse "estabilizada no ecrã" (i.e., alvo) pressionando a tecla "S" caso esta fosse negativa e a tecla " $L$ " caso fosse positiva. O estudo iniciava-se com a realização de 30 ensaios de treino de rapidez de resposta (nos quais se dava feedback para responder abaixo dos $1500 \mathrm{~ms}$ ), seguindo a fase de primação. Cada ensaio era iniciado por um ecrã que exibia um sinal de "+" (500ms) para focalizar a atenção do participante. A palavra-primo era apresentada no centro do ecrã durante $200 \mathrm{~ms}$ seguindo-se um ecrã em branco (100ms), findo o qual aparecia a palavra-alvo, que permanecia visível até que uma tecla de resposta fosse pressionada ${ }^{7}$. O intervalo entre ensaios era de quatro segundos.

Finda a fase de primação (constituída por 30 ensaios), os participantes respondiam ainda a uma série de questões de controlo, incluindo uma detecção de eventuais suspeitas face ao procedimento de primação ${ }^{8}$.

No final da experiência agradecia-se a colaboração dos participantes e respondia-se às questões colocadas.

\section{Resultados e discussão}

Tempos de Resposta: As médias dos tempos de resposta aos estímulos-alvo positivos e negativos nas condições congruentes e incongruentes foram integradas no modelo ANOVA de medidas repetidas definido pelo delineamento após transformação logarítmica de base "e" (ln). Replicando os dados da literatura, verificou-se a facilitação do desempenho nos ensaios em que ambas as palavras partilham a mesma valência (i.e., são congruentes em termos avaliativos, $M=990 \mathrm{~ms}$, d.p. $=573$ ) comparativamente aos ensaios incongruentes $(M=1149 \mathrm{~ms}, d . p .=467 ; F(1,58)=16,30, p<0,001)$. A valência do estímulo-primo parece porém interferir com este efeito (ver Figura 1). $\mathrm{O}$ efeito da interacção global não atinge níveis de significância estatísticos que nos esclareçam quanto à sua presença ou ausência $(F(1,58)=2,72$, $p=0,104)$. Na realidade, apesar do padrão das médias sugerir que o efeito

\footnotetext{
${ }^{7}$ Este intervalo entre o início da apresentação do estímulo-primo e o início da apresentação do alvo denomina-se S.O.A. (i.e., Stimulus Onset Asynchrony, para revisão ver Hermans, De Houwer, \& Eelen, 2001), constituindo um aspecto metodológico central nos paradigmas de primação (ver também Bargh \& Chartrand, 2000). No presente estudo, o S.O.A. utilizado foi de $300 \mathrm{~ms}$.

${ }^{8}$ Dado que nenhum participante revelou suspeitas quanto às hipóteses em estudo, os dados verificados nestas questões não serão doravante mencionados.
} 
possa ser mais forte relativamente a items positivos, contrastes planeados sugerem que a vantagem da congruência está patente para ambas as valências dos primos. É apenas mais claro o efeito para as palavras positivas $(t(58)=4,38, p<0,001)$, do que para as negativas $(t(58)=2,00, p=0,025)$. Futuros estudos terão de nos esclarecer deste fenómeno.

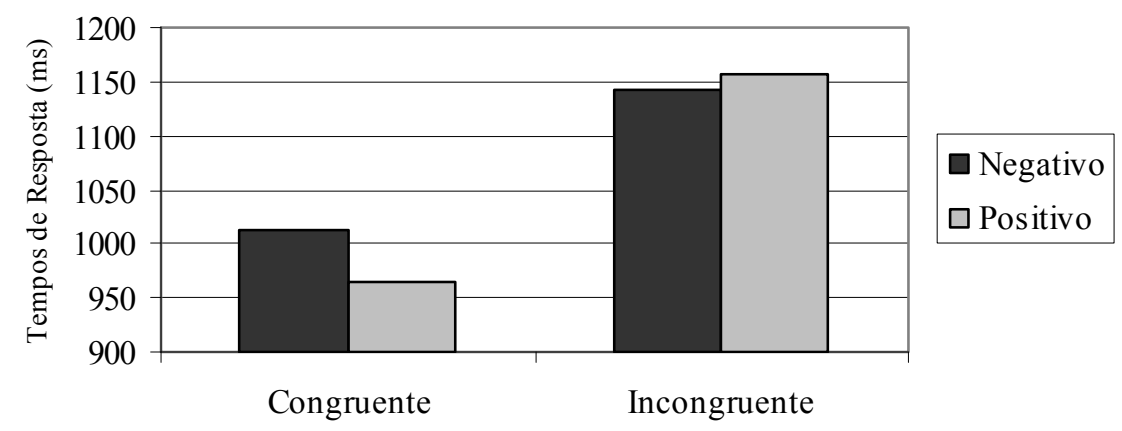

Alvo

Figura 1. Interacção entre tipo de ensaio e valência do estímulo-primo.

Proporção de acertos: Como se pretende argumentar no presente artigo, o desempenho pode igualmente traduzir os efeitos de primação neste tipo de paradigma. Tal reflectiria uma maior percentagem de acertos aquando de congruência avaliativa. Para testar esta hipótese, realizou-se uma ANOVA de medidas repetidas na proporção de acertos associados a estímulos positivos e negativos em condições de congruência e incongruência. Note-se que, por "acerto", se compreende a resposta correcta à valência do alvo, independentemente da valência primada. Esta análise suporta a nossa hipótese ao sugerir que a proporção de acertos é mais elevada quando os estímulos são congruentes $(M=0,8, d . p .=0,21)$ do que quando são incongruentes $(M=0,5$, d.p. $=0,33 ; F(1,58)=24,55, p<0,001)$. Adicionalmente, detectou-se também um efeito principal da valência do estímulo-primo $(F(1,58)=4,28$, $p=0,043)$, patente na Figura 2, que indica uma maior proporção de acertos quando o primo é positivo, independentemente do tipo de ensaio.

Os dados deste experimento sugerem claramente a existência do efeito típico deste tipo de paradigma: facilitação do desempenho (respostas mais rápidas) aquando de congruência avaliativa. Adicionalmente, os dados reflectem uma interferência no padrão do número de respostas correctas. A incongruência afectiva permite identificar a interferência do estímulo-primo 
que contraria a percepção directa do estímulo, induzindo respostas erradas (e, por conseguinte, reduzindo o número de acertos). Demonstramos, assim, a possível identificação de efeitos de influência (associados à natureza de resposta) em paradigmas de interferência. No experimento que se segue, procuraremos identificar efeitos ao nível dos tempos de resposta num paradigma de influência.

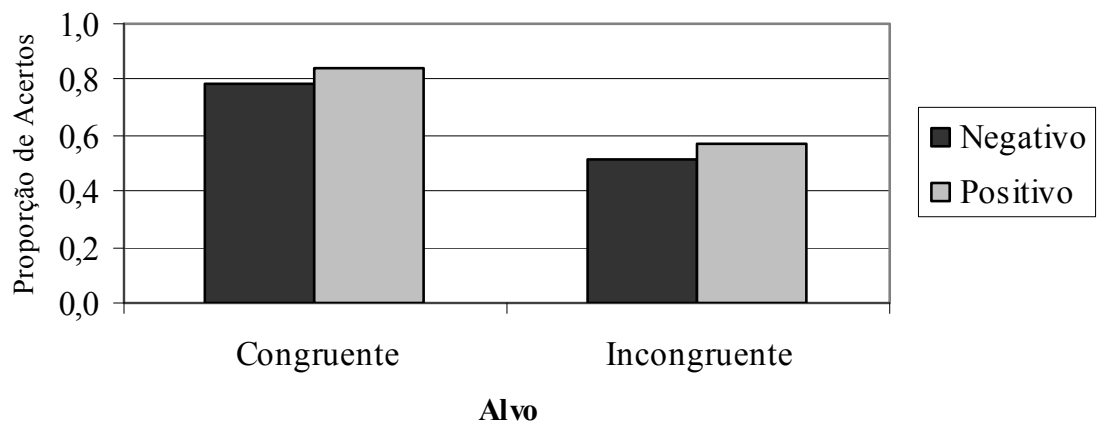

Figura 2. Interacção entre tipo de ensaio e valência do estímulo-primo.

\section{Experimento 2}

Este experimento tem por base o paradigma desenvolvido por Murphy e Zajonc (1993), onde se demonstra que a utilização de fotografias de faces com uma expressão zangada ou feliz, enquanto estímulos-primo, influenciava a avaliação dos estímulos subsequentes, no sentido da valência activada (i.e., negativa ou positiva, respectivamente). Os autores apenas detectaram este efeito quando a apresentação do estímulo-primo era efectuada a nível subliminar, mas não a nível supraliminar, o que se poderá dever à extensa duração nesta última $(1000 \mathrm{~ms})$ e à natureza do material. Assim, em vez de fotografias com expressões faciais correspondentes a emoções específicas (primos) e caracteres chineses (alvos), utilizámos um conjunto de imagens de diferentes categorias, pré-testadas quanto à sua valência (Prada \& Garcia-Marques, 2006), comparando ainda os resultados obtidos com ambas as formas de primação. Testámos, então, os efeitos de influência na avaliação de alvos constituídos por fotografias de objectos do quotidiano (e não meros símbolos ambíguos e novos para os participantes), procurando analisar os seus efeitos em termos de tempos de respostas. Por outras palavras, focámos a possibilidade de as avaliações destes objectos, previamente avaliados como neutros, serem determinadas no sentido da 
valência activada pelo primo), explorando igualmente o tempo que os participantes demoram a realizar tais julgamentos.

\section{Participantes e Delineamento Experimental}

Participaram voluntariamente, mediante crédito parcial numa disciplina do curso de Psicologia, 41 estudantes $(73,2 \%$ do género feminino; $\mathrm{M}_{\mathrm{idade}}=20,1$ anos, d.p. $\left.=2,4\right)$, distribuídos aleatoriamente pelos dois grupos experimentais definidos pelo primeiro factor do delineamento: 2 (Primação: supraliminar; subliminar) x 3 (Valência do estímulo-primo: negativo, neutro, positivo). O segundo factor é intra-participantes.

\section{Material}

O conjunto de estímulos é constituído por imagens seleccionadas do Ficheiro de Imagens Multicategoriais (F.I.M., Prada \& Garcia-Marques, 2006). Especificamente, os estímulos-primo consistem em 12 imagens $^{9}$ avaliadas como negativas $\left(M_{\text {Valência }}=3,0, d . p .=0,11\right)$, neutras $\left(M_{\text {Valên- }}\right.$ cia $=5,1, d . p .=0,13)$ e positivas $\left(M_{\text {Valência }}=7,1, d . p .=0,10\right)$. O conjunto de estímulos-alvo, por sua vez, é composto por 12 imagens de objectos do quotidiano pré-testadas como neutras $\left(M_{\text {Valência }}=5,0, d \cdot p .=0,29\right)^{10}$.

Utilizou-se ainda um conjunto adicional de estímulos para os ensaios distractivos, que, por sua vez, era composto por 24 imagens de outros tipos de objectos avaliados como neutros (e.g., objectos domésticos como "garrafa", "cabide" ou "molas de roupa"; $M_{\text {Valência }}=5,1, d . p$. $=0,21$ ).

\section{Procedimento}

Os participantes foram convidados a colaborar num alegado estudo sobre "Identificação e avaliação de imagens, em contextos simples e complexos", realizado com suporte informático (programa E-prime). Estes eram informados de que seriam expostos a sequências de imagens, apresentadas durante breves instantes, sendo-lhes pedido que avaliassem de forma rápida e espontânea a imagem que ficasse estabilizada no ecrã, recorrendo a uma escala de 5 pontos ( $1=$ Não gosto nada; $5=$ Gosto muito).

9 O sub-conjunto de estímulos negativos é composto por imagens de "arame farpado", "mão sangrenta", "mosca" e "cama de hospital", o de estímulos neutros por imagens de "esponja", "relógio", "rolo de cordel" e "lata" e o de estímulos positivos por imagens de "guitarra", "borboleta", "brinde" e "hipopótamo". Nessa ordem, as imagens correspondem aos seguintes números do FIM (Prada \& Garcia-Marques, 2006): 11, 12, 13 e 15; 45, $51,53$ e $57 ; 161,163,166$ e 167$)$

$10 \mathrm{O}$ conjunto de estímulos-alvo era constituído por imagens de objectos do quotidiano como ferramentas e material de escritório (números 39, 41, 43, 44, 47, 49, 50, 54, 56, 67, 72 e 93 do FIM). 
$\mathrm{Na}$ condição de primação supraliminar, cada ensaio era iniciado pela apresentação de um sinal “+”(500ms) como forma de focalizar a atenção do participante, seguido de um ecrã em branco $(500 \mathrm{~ms})$. Posto isto, apresentava-se o estímulo-primo $(200 \mathrm{~ms})$, novamente um ecrã em branco $(500 \mathrm{~ms})$, e por último apresentava-se o alvo $(S . O . A .=700 \mathrm{~ms})$ até que uma tecla de resposta fosse pressionada. A condição subliminar era semelhante a esta, exceptuando o tempo de apresentação dos primos $\left(17 \mathrm{~ms}^{11}\right)$ e o intervalo decorrente até à apresentação do alvo $(50 \mathrm{~ms} ; S . O . A .=67 \mathrm{~ms})$.

Os ensaios distractivos, por sua vez, tinham como mero objectivo confirmar o alegado objectivo do estudo, consistindo na avaliação de imagens, pré-testadas como neutras, quer de forma individualizada (i.e., apenas uma imagem, à semelhança dos ensaios críticos da condição de controlo), quer em sequências de três imagens (em que apenas a terceira seria alvo de avaliação).

No final de cada ensaio era introduzida uma tarefa "distractiva" (com vista a reduzir o impacto de um ensaio no subsequente) onde era pedido a cada participante que memorizasse e reproduzisse um número composto por nove dígitos. Após 24 ensaios de avaliação de imagens (12 ensaios críticos), os participantes realizavam uma última tarefa destinada a confirmar a eficácia da manipulação da apresentação subliminar dos estímulos. Por fim, agradecia-se a participação no estudo e respondia-se a eventuais questões acerca do mesmo.

\section{Resultados e discussão}

Julgamentos Avaliativo: Com o objectivo de testar a replicação dos efeitos típicos de influência, as médias das respostas avaliativas às imagens-alvo foram integradas no modelo ANOVA misto onde os diferentes grupos sujeitos a uma primação supra ou subliminar foram comparados nas respostas dadas associadas às três valências de primos. Contrastes planeados associados a este modelo permitiram testar directamente a hipótese de influência do estímulo-primo.

De acordo com o impacto esperado da valência dos estímulos primos na avaliação dos estímulos alvo, verificou-se a existência de um efeito principal deste factor $(F(2,78)=14,80, p<0,001)$, obtendo-se avaliações mais positivas nos alvos primados com estímulos positivos $(M=3,03$, d.p. $=0,69)$ do que naqueles primados com estímulos neutros $(M=2,87$, d.p. $=0,72)$ ou negativos $(M=2,45, d . p .=0,83)$.

11 A opção por esta duração de apresentação prende-se com a recomendação de durações suficientemente curtas para a maioria dos participantes (ver Bargh \& Chartrand, 2000). De facto, tal é o tempo mínimo permitido pelos equipamentos utilizados (devido ao refresh rate dos ecrãs). 


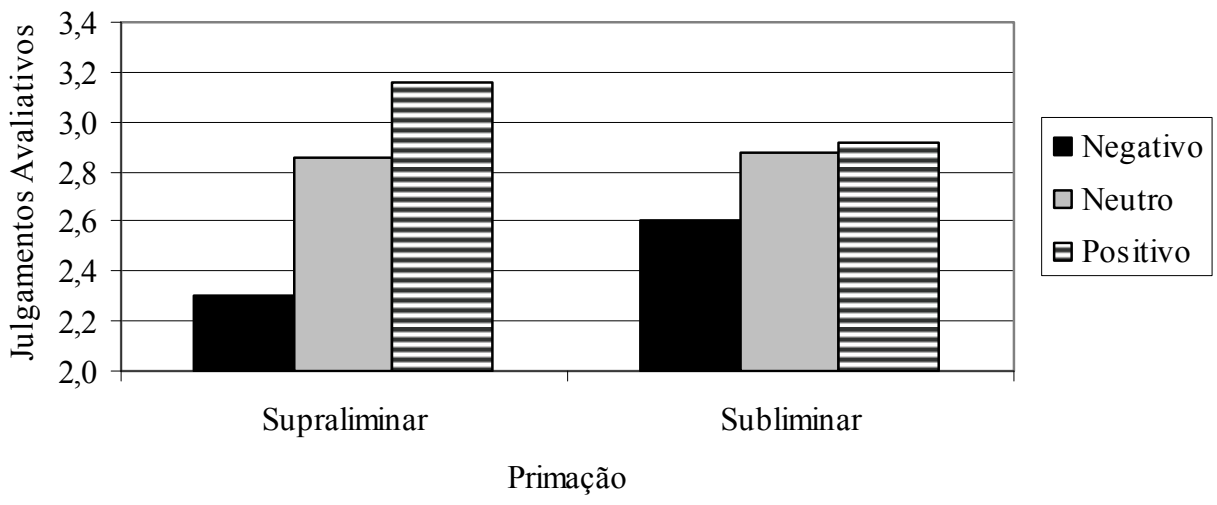

Figura 3. Interacção entre valência do estímulo-primo e condição experimental

A tendência linear associada ao efeito das três valências ao nível dos julgamentos revelou-se significativa, quer as apresentações fossem supraliminares $(t(39)=4,79, p<0,001$, unilateral) ou subliminares $(t(39)=1,74$, $p=0,045$, unilateral). Contudo, como pode ser observado na Figura 3, na condição subliminar não se distingue entre as avaliações verificadas quando os estímulos são primados com estímulos neutros ou quando estes são positivos $(t(39)<1)$. Estes resultados parecem sugerir a possibilidade de que apenas os estímulos negativos exerceram o impacto esperado a nível subliminar.

Tempos de Resposta: Analisaram-se os tempos de resposta (após transformação logarítmica $\ln$ ) associados às respostas subsequentes aos três tipos de primos (medidas repetidas) em ambos os grupos, por uma ANOVA mista.

A valência do estímulo-primo parece influenciar a rapidez de resposta aos alvos $(F(2,78)=12,15, p<0,001)$, verificando-se avaliações mais rápidas nos alvos primados com estímulos neutros $(M=2658 \mathrm{~ms}$ d.p. $=1036)$ do que naqueles primados com estímulos valenciados $\left(M_{\text {negativos }}=2941 \mathrm{~ms}\right.$, d.p. $\left.=1030 ; M_{\text {positivos }}=3479 \mathrm{~ms}, d . p .=1197\right)(t(39)=3,60, p<0,001) . \mathrm{De}$ facto, considerando apenas os alvos primados com estímulos valenciados, os positivos são os que exibem tempos de resposta superiores $(t(39)=3,28$, $p=0,002)$. Este efeito não é qualificado pelo tipo de primação efectuado $(F(2,78)<1)$ mas, como patente na Figura 4, na condição subliminar os estímulos-primo negativos não se diferenciam dos neutros $(t(39)<1)$. 


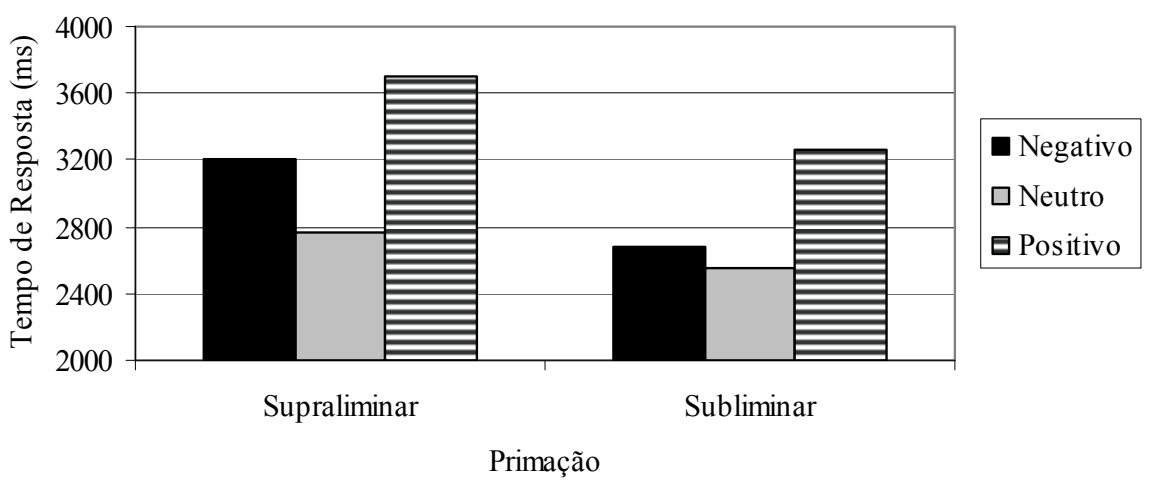

Figura 4. Interacção entre valência do estímulo-primo e condição experimental.

Estes dados sugerem que a valência dos estímulos primos, quer apresentados consciente quer inconscientemente, interfere no tempo que os indivíduos levam a realizar os seus julgamentos. Quer estes sejam positivos quer negativos, os indivíduos parecem levar mais tempo a realizar o julgamento do que se não sofrerem qualquer influência do contexto (em termos de activação de valência). Esta interferência parece ser maior quando o primo é positivo.

Efectuou-se ainda uma análise exploratória na qual dicotomizámos as respostas dadas pelos participantes de forma a analisar o padrão de facilitação de desempenho verificado no paradigma de interferência (Experimento 1). Ou seja, considerou-se como "ensaios congruentes" os ensaios em que o primo era negativo (ou positivo) e a resposta na escala era igual a " 1 " ou "2" (ou "4" ou "5"). Apesar das limitações inerentes à realização desta análise ${ }^{12}$, apurou-se que, quando os primos eram apresentados supraliminarmente, as respostas pareciam ser facilitadas (sendo a diferença de $227 \mathrm{~ms}$ ) aquando de congruência $(M=3195 \mathrm{~ms}, d . p .=1819)$ comparativamente à incongruência $(M=3422 \mathrm{~ms}, d . p .=1544)$. Já ao nível da primação subliminar, tal diferença é menos evidente $\left(53 \mathrm{~ms} ; M_{\text {congruente }}=2675 \mathrm{~ms}, d . p .=1390\right.$; $\left.M_{\text {incongruente }}=2710 \mathrm{~ms}, d . p .=2729, d . p .=1827\right)$.

12 Por limitações entende-se o facto de, no presente experimento, se ter utilizado uma escala de 5 pontos, fazendo com que, para a análise de congruência/incongruência, se tenha que excluir as respostas neutras (i.e., iguais a “3”). Tal opção faz com que algumas células possam ficar em branco o que, por sua vez, prejudica a análise de médias. À luz destas limitações, a análise que acima se apresenta deve ser interpretada apenas como um indicador. 


\section{Discussão Geral}

Neste artigo, procurámos definir o fenómeno de primação afectiva e clarificar as diferenças entre os dois procedimentos mais usuais para a sua investigação. Os experimentos apresentados visavam não só ilustrar tais paradigmas e os padrões de resultados tipicamente encontrados, mas também explorar variáveis adicionais em cada um destes. O primeiro estudo consiste numa adaptação do que denominámos por paradigma de interferência (Fazio et al., 1986) e permitiu demonstrar a facilitação do desempenho quando ambos os estímulos de um dado ensaio partilham a mesma valência afectiva comparativamente a quando são incongruentes. Para além deste efeito, detectado através da análise dos tempos de resposta, analisou-se também a proporção de acertos (i.e., respostas correctas à valência do alvo) observados. Verificámos que, em caso de ensaios congruentes, se verifica um menor número de erros. O segundo estudo, por sua vez, teve por base o paradigma de influência (Murphy \& Zajonc, 1993) e permitiu demonstrar o impacto da activação de uma valência prévia no julgamento de um alvo anteriormente percebido como neutro. Ou seja, neste caso, a variável fundamental a considerar é o próprio julgamento avaliativo do alvo. Neste estudo explorámos também a possibilidade da valência activada influenciar a velocidade com que os julgamentos do alvo eram efectuados. De facto, os alvos primados com estímulos negativos exibem tempos de resposta inferiores aos verificados para os que são primados positivamente. Este padrão está de acordo com a prioridade atencional, considerada adaptativa, atribuída aos estímulos negativos (ver Unkelbach, Fiedler, Bayer, Stegmuller, \& Danner, 2008). Contudo, como mencionado, a análise mais adequada seria a de comparar os tempos médios de resposta em situação de congruência com os verificados em incongruência. Tal análise é dificultada quando a variável dependente (julgamento do alvo) é medida com recurso a uma escala como a que utilizámos (i.e., cinco pontos). Uma possibilidade seria a de utilizar escalas com pontos em número par (e.g., quatro pontos) de forma a possibilitar uma dicotomização adequada. No entanto, pode argumentar-se que, neste caso, retirando o ponto médio da escala, se estaria a forçar a extremar a avaliação de um objecto que, para o indivíduo, é percebido como neutro. Outra possibilidade de investigar o impacto da (in)congruência, seria a de utilizar estímulos-alvo moderadamente positivos ou negativos, de forma a diferenciá-los. Em suma, enquanto que a investigação de efeitos de influência (associados à natureza de resposta) em paradigmas de interferência não é problemática do ponto de vista metodológico, a identificação de efeitos ao nível dos tempos de resposta num paradigma de influência apresenta mais constrangimentos. 
Sublinhamos ainda que os paradigmas de primação afectiva aqui apresentados se referem apenas ao estudo do impacto da activação de uma dada valência no processamento. Ou seja, trata-se da conceptualização do termo "afecto" no seu sentido mais lato. Mesmo nos estudos em que se utilizam imagens de expressões faciais associadas a emoções (e.g., Murphy \& Zajonc, 1993; Monahan, 1998), pressupõe-se que a propriedade relevante activada é a percepção destas como boas ou más, positivas ou negativas (para revisão da distinção entre afecto e emoção, ver Garcia-Marques, 2001). Contudo, estes paradigmas são passíveis de ser adaptados ao objectivo de estudar o impacto de emoções específicas (e.g., Pell, 2005). Por exemplo, Small, Lerner e Fischhoff (2006) demonstraram que a natureza específica da emoção negativa primada (tristeza ou raiva) determinava as atribuições realizadas acerca de um evento trágico (e.g., 11 de Setembro).

\section{Aplicabilidade e relevância do fenómeno de Primação Afectiva}

Neste artigo, abordamos o fenómeno de primação afectiva pelo seu interesse para o desenvolvimento de uma maior compreensão dos processos cognitivos que subjazem os nossos julgamentos. Existe, porém, uma outra faceta deste fenómeno que convém aqui salientar - a possibilidade de identificação de atitudes implícitas de indivíduos.

Apesar da variabilidade encontrada na literatura quanto à operacionalização do constructo de atitude, existe algum consenso relativamente à existência de uma componente avaliativa na estrutura de uma atitude (para revisão ver Petty, Briñol, \& DeMarree, 2007). O facto de os paradigmas de primação afectiva assentarem nesta componente torna-os ferramentas úteis para a medição implícita de atitudes (para revisão ver Fazio \& Olsen, 2003).

Como mencionado, num paradigma de interferência, um dado objecto atitudinal pode ser emparelhado com um alvo de valência positiva ou negativa. Imaginemos um ensaio em que o estímulo-primo "Bush" precede um dos seguintes alvos: "festa" (em termos normativos é positivo) ou "cobra" (em termos normativos é negativo). Assim, espera-se que as pessoas que possuem uma atitude mais favorável face a Bush respondam mais rapidamente (i.e., facilitação de desempenho) a "festa" do que, por exemplo, a "cobra" (pois existiria incongruência afectiva). Contrariamente, as que assumem uma atitude mais desfavorável face a este político norte-americano deverão exibir o padrão inverso (i.e., respostas mais rápidas a "cobra" do que a "festa"). Fazio, Jackson, Dunton e Williams (1995) aplicaram um procedimento deste tipo enquanto medida de atitudes raciais. Especificamente, a tarefa dos participantes consistia apenas em indicar, o mais rapidamente possível, se a conotação de um adjectivo-alvo (e.g., "agradável" ou "horrível") era negativa ou positiva. Previamente a cada avaliação, 
era apresentada uma fotografia de um estudante branco ou negro. O padrão de resultados sugere a activação automática de reacções negativas face aos negros dado que as fotografias destes, comparativamente às de brancos, facilitavam a resposta aos adjectivos negativos e interferiam com a resposta aos positivos. Note-se que, subjacente a uma das mais notórias medidas implícitas - Teste de Atitude Implícita (Implicit Association Test - IAT, Greenwald, McGhee, \& Schwartz, 1998), está também uma lógica de interferência (para revisão ver Fazio \& Olsen, 2003).

Também o paradigma desenvolvido por Murphy e Zajonc (1993) foi adaptado recentemente, por Payne, Cheng, Govorun e Stewart (2005), enquanto medida implícita de atitudes - Procedimento de Atribuição Enviesada (Affect Misattribution Procedure ou AMP). Também neste procedimento, o julgamento avaliativo de um alvo é precedido pela apresentação de um objecto atitudinal (e.g., "George W. Bush"). Contudo, neste caso, os alvos são neutros ou ambíguos (e.g., símbolo abstracto) em vez de claramente valenciados. Ainda que as instruções indiquem que apenas o símbolo deve ser avaliado, se as pessoas atribuírem enviesadamente as reacções suscitadas pelo objecto atitudinal ao alvo, serão as reacções face ao estímulo-primo a enviesar as avaliações do símbolo. Ou seja, se este induz uma influência negativa nos julgamentos do alvo, poder-se-á inferir que a pessoa possui uma atitude negativa face a Bush.

Esta relevância funcional dos paradigmas de primação afectiva torna cada vez mais premente uma completa compreensão dos diferentes paradigmas que permitem operacionalizar o conceito de atitude implícita. Ambos os tipos de efeitos contextuais no processamento são relevantes do ponto de vista prático, dado permitirem esta mensuração. Como mencionado, tanto a facilitação de desempenho entre um par de estímulos como a verificação de uma avaliação mais positiva após a exposição a um estímulo com essa valência, poderão ser interpretados como atitudes favoráveis face a um dado objecto atitudinal.

Neste artigo, apesar de termos contrastado dois paradigmas, não os abordamos relativamente à sua sensibilidade e validade enquanto instrumentos de mensuração. A utilização destes num delineamento intra-participantes poderá elucidar-nos futuramente acerca de tais características. Os nossos dados, até ao momento, permitem, no entanto, salientar as vantagens do paradigma de interferência na sua capacidade de fornecer duas medidas complementares, passíveis de análise com significado avaliativo: a natureza do julgamento e os tempos de latência. 


\section{Referências}

Abrams, R. L., Klinger, M. R., \& Greenwald, A. G. (2002). Subliminal words activate semantic categories (not automated motor responses). Psychonomic Bulletin \& Review, 9(1), 100-106.

Balota, D. A., \& Lorch, R. F. (1986). Depth of automatic spreading activation: Mediated priming effects in pronunciation but not in lexical decision. Journal of Experimental Psychology: Learning, Memory, and Cognition, 12(3), 336-345.

Banse, R. (1999). Automatic evaluation of self and significant others: Affective priming in close relationships. Journal of Social and Personal Relationships, 16(6), 803-821.

Banse, R. (2001). Affective priming with liked and disliked persons: Prime visibility determines congruency and incongruency effects. Cognition \& Emotion, 15(4), 501-520.

Banse, R. (2003). Beyond verbal self-report: Priming methods in relationship research. In J. Musch \& K. C. Klauer (Eds.), The psychology of evaluation: Affective processes in cognition and emotion. (pp. 245-274). Mahwah, NJ, US: Lawrence Erlbaum Associates Publishers.

Bargh, J. A. (1994). The four horsemen of automaticity: Awareness, intention, efficiency, and control in social cognition. In R. S. Wyer, Jr. \& T. K. Srull (Eds.), Handbook of social cognition, Vol. 1: Basic processes (2nd ed.) (pp. 1-40). Hillsdale: Lawrence Erlbaum Associates, Inc.

Bargh, J. A. (2006). Agenda 2006: What have we been priming all these years? On the development, mechanisms, and ecology of nonconscious social behavior. European Journal of Social Psychology, 36(2), 147-168.

Bargh, J. A., Chaiken, S., Govender, R., \& Pratto, F. (1992). The generality of the automatic attitude activation effect. Journal of Personality and Social Psychology, 62(6), 893-912.

Bargh, J. A., \& Chartrand, T. L. (2000). The mind in the middle: A practical guide to priming and automaticity research. In H. T. Reis \& C. M. Judd (Eds.), Handbook of research methods in social and personality psychology (pp. 253-285). Cambridge University Press.

Chaiken, S., \& Bargh, J. A. (1993). Occurrence versus moderation of the automatic attitude activation effect: Reply to Fazio. Journal of Personality and Social Psychology, 64(5), 759-765.

Chan, E., Ybarra, O., \& Schwarz, N. (2006). Reversing the affective congruency effect: The role of target word frequency of occurrence. Journal of Experimental Social Psychology, 42(3), 365-372.

De Houwer, J., \& Hermans, D. (2001). Automatic affective processing. Cognition \& Emotion, pp. 113-114.

De Houwer, J., Hermans, D., \& Eelen, P. (1998). Affective and identity priming with episodically associated stimuli. Cognition \& Emotion, 12(2), 145-169.

De Houwer, J., \& Randell, T. (2002). Attention to primes modulates affective priming of pronunciation responses. Experimental Psychology, 49(3), 163-170. 
De Houwer, J., \& Randell, T. (2004). Robust affective priming effects in a conditional pronunciation task: Evidence for the semantic representation of evaluative information. Cognition \& Emotion, 18(2), 251-264.

Draine, S. C., \& Greenwald, A. G. (1998). Replicable unconscious semantic priming. Journal of Experimental Psychology: General, 127(3), 286-303.

Duckworth, K. L., Bargh, J. A., Garcia, M., \& Chaiken, S. (2002). The automatic evaluation of novel stimuli. Psychological Science, 13(6), 513-519.

Fazio, R. H. (2001). On the automatic activation of associated evaluations: An overview. Cognition \& Emotion, 15(2), 115-141.

Fazio, R. H., Jackson, J. R., Dunton, B. C., \& Williams, C. J. (1995). Variability in automatic activation as an unobstrusive measure of racial attitudes: A bona fide pipeline? Journal of Personality and Social Psychology, 69(6), 1013-1027.

Fazio, R. H., \& Olson, M. A. (2003). Implicit measures in social cognition research: Their meaning and uses. Annual Review of Psychology, 54, 297-327.

Fazio, R. H., Sanbonmatsu, D. M., Powell, M. C., \& Kardes, F. R. (1986). On the automatic activation of attitudes. Journal of Personality and Social Psychology, 50(2), 229-238.

Ferguson, M. J., \& Bargh, J. A. (2003). The constructive nature of automatic evaluation. In J. Musch \& K. C. Klauer (Eds.), The psychology of evaluation: Affective processes in cognition and emotion. (pp. 169-188). Mahwah, NJ, US: Lawrence Erlbaum Associates Publishers.

Fiedler, K. (2003). The hidden vicissitudes of the priming paradigm in evaluative judgment research. In J. Musch \& K. C. Klauer (Eds.), The psychology of evaluation: Affective processes in cognition and emotion. (pp. 109-137). Mahwah, NJ, US: Lawrence Erlbaum Associates Publishers.

Garcia-Marques, T. (2001). A dimensão afectiva: distinguindo afecto, emoção, estado de espírito e sentimento. Psicologia: Teoria, Investigação e Prática, 6(2), 253-268.

Garcia-Marques, T. (2005). Diferenciando "primação afectiva" de "primação cognitiva”. Análise Psicológica, 4(XXIII), 437-447.

Gawronski, B., Deutsch, R., LeBel, E. P., \& Peters, K. R. (2008). Response interference as a mechanism underlying implicit measures: Some traps and gaps in the assessment of mental associations with experimental paradigms. European Journal of Psychological Assessment, 24(4), 218-225.

Greenwald, A. G., McGhee, D. E., \& Schwartz, J. L. K. (1998). Measuring individual differences in implicit cognition: The Implicit Association Test. Journal of Personality and Social Psychology, 74(6), 1464-1480.

Henik, A., Nissimov, E., Priel, B., \& Umansky, R. (1995). Effects of cognitive load on semantic priming in patients with schizophrenia. Journal of Abnormal Psychology, 104(4), 576-584.

Hermans, D., De Houwer, J., \& Eelen, P. (2001). A time course analysis of the affective priming effect. Cognition \& Emotion, 15(2), 143-165.

Hermans, D., Smeesters, D., De Houwer, J., \& Eelen, P. (2002). Affective priming for associatively unrelated primes and targets. Psychologica Belgica, 42(3), 191-212. 
Klauer, K. C. (1998). Affective priming. European Review of Social Psychology, 8, 67-103.

Klauer, K. C., \& Musch, J. (2003). Affective priming: Findings and theories. In J. Musch \& K. C. Klauer (Eds.), The psychology of evaluation: Affective processes in cognition and emotion. (pp. 7-49). Mahwah, NJ, US: Lawrence Erlbaum Associates Publishers.

Klauer, K. C., Roßnagel, C., \& Musch, J. (1997). List-context effects in evaluative priming. Journal of Experimental Psychology: Learning, Memory, and Cognition, 23(1), 246-255.

Meyer, D. E., \& Schvaneveldt, R. W. (1971). Facilitation in recognizing pairs of words: Evidence of a dependence between retrieval operations. Journal of Experimental Psychology, 90(2), 227-234.

Monahan, J. L. (1998). I don't know it but I like you: The influence of nonconscious affect on person perception. Human Communication Research, 24(4), 480-500.

Murphy, S. T., \& Zajonc, R. B. (1993). Affect, cognition, and awareness: Affective priming with optimal and suboptimal stimulus exposures. Journal of Personality and Social Psychology, 64(5), 723-739.

Neely, J. H. (1976). Semantic priming and retrieval from lexical memory: Evidence for facilitatory and inhibitory processes. Memory \& Cognition, 4, 648-654.

Neely, J. H. (1977). Semantic priming and retrieval from lexical memory: Roles of inhibitionless spreading activation and limited-capacity attention. Journal of Experimental Psychology: General, 106(3), 226-254.

Neely, J. H., Besner, D., \& Humphreys, G. W. (1991). Semantic priming effects in visual word recognition: A selective review of current findings and theories. In Basic processes in reading: Visual word recognition. (pp. 264-336). Hillsdale, NJ, England: Lawrence Erlbaum Associates, Inc.

Payne, B. K., Cheng, C. M., Govorun, O., \& Stewart, B. D. (2005). An inkblot for attitudes: Affect misattribution as implicit measurement. Journal of Personality and Social Psychology, 89(3), 277-293.

Pell, M. D. (2005). Nonverbal emotion priming: Evidence from the 'facial affect decision task'. Journal of Nonverbal Behavior, 29(1), 45-73.

Petty, R. E., Briñol, P., \& DeMarree, K. G. (2007). The Meta-Cognitive Model (MCM) of attitudes: Implications for attitude measurement, change, and strength. Social Cognition, 25(5), 657-686.

Prada, M., \& Garcia-Marques, T. (2006a). Familiaridade e Primação Afectiva. Paper presented at the VI Simpósio Nacional de Investigação em Psicologia, Évora, Portugal.

Prada, M., \& Garcia-Marques, T. (2006b). Normas da valência das imagens do Ficheiro de Imagens Multicategoriais (FIM). Laboratório de Psicologia, 4(1), 109-137.

Prada, M., \& Silva, R. (2008). De triunfante a terrível: Avaliação de valência e familiaridade de adjectivos em língua Portuguesa. Laboratório de Psicologia, 6(1), 25-47. 
Simon, J. R., \& Berbaum, K. (1990). Effect of conflicting cues on information processing: The 'Stroop effect' vs. the 'Simon effect.' Acta Psychologica, 73(2), 159-170.

Small, D. A., Lerner, J. S., \& Fischhoff, B. (2006). Emotion priming and attributions for terrorism: Americans' reactions in a national field experiment. Political Psychology, 27(2), 289-298.

Spruyt, A., Hermans, D., De Houwer, J., \& Eelen, P. (2002). On the nature of the affective priming effect: Affective priming of naming responses. Social Cognition, 20(3), 227-256.

Unkelbach, C., Fiedler, K., Bayer, M., Stegmuller, M., \& Danner, D. (2008). Why positive information is processed faster: The density hypothesis. Journal of Personality and Social Psychology, 95(1), 36-49.

Wentura, D. (1998). Affective priming in the lexical decision task: Evidence for post-lexical judgemental tendencies. Sprache \& Kognition, 17(3), 125-137.

Wentura, D. (1999). Activation and inhibition of affective information: Evidence for negative priming in the evaluation task. Cognition \& Emotion, 13(1), 65-91.

Winkielman, P., Schwarz, N., Fazendeiro, T. A., \& Reber, R. (2003). The hedonic marking of processing fluency: Implications for evaluative judgment. In $\mathrm{J}$. Musch \& K. C. Klauer (Eds.), The psychology of evaluation: Affective processes in cognition and emotion. (pp. 189-217). Mahwah, NJ, US: Lawrence Erlbaum Associates Publishers.

Wittenbrink, B. (2007). Measuring attitudes through priming. In B. Wittenbrink \& N. Schwarz (Eds.), Implicit measures of attitudes (pp. 17-58). New York, NY, US: Guilford Press. 